\title{
Touch-down and take-off hysteresis of magnetic recording sliders on discrete track media
}

\author{
Y. Yoon · F. E. Talke
}

Received: 31 July 2008/Accepted: 13 April 2009/Published online: 26 April 2009

(C) The Author(s) 2009. This article is published with open access at Springerlink.com

\begin{abstract}
The touch-down and take-off characteristics of a typical pico-type magnetic recording slider is investigated as a function of pressure level and groove dimensions of discrete track recording (DTR) media. Keeping the ambient pressure constant, we found that the touch-down velocity was higher for DTR disks than for "smooth" disks without discrete tracks. Likewise, the "ambient" touchdown pressure at constant velocity was found to be higher for DTR disks than for smooth media. The hysteresis between touch-down and take-off velocity and touch-down and take-off ambient pressure was found to be larger for DTR media than for smooth media. Start/stop tests on discrete track media were performed to investigate the effect of grooves of discrete track media on the tribology of the head/disk interface.
\end{abstract}

\section{Introduction}

In order to achieve areal densities in hard disk drives (HDD) on the order of $1 \mathrm{Tbit} / \mathrm{in}^{2}$, discrete track recording (DTR) and bit patterned media (BPM) are presently being investigated. DTR media reduce magnetic "cross-talk" in the radial direction by physically separating adjacent recording tracks from each other (Wachenschwanz et al. 2005). BPM feature individual islands for each bit, thereby

Y. Yoon $(\bowtie) \cdot$ F. E. Talke

Center for Magnetic Recording Research,

U. C. San Diego, La Jolla, CA 92093-0401, USA

e-mail: paul@talkelab.ucsd.edu reducing cross talk for both the radial and the circumferential direction.

The flying behavior of a slider over discrete track media is affected by the presence of the grooves on the disk. In particular, the steady state flying height of a slider flying over discrete track media is lower than that of a slider flying over smooth media due to the loss of air bearing pressure over the grooves. Numerical investigations by Duwensee et al. $(2006,2007)$ have shown that the "flying height loss" $\Delta \mathrm{h}$ of a slider over discrete track media can be determined by

$\Delta h=d \cdot \frac{w}{p}$,

where $d$ is the groove depth, $w$ is the groove width, and $p$ is the track pitch.

To achieve an areal density of $1 \mathrm{Tbit} / \mathrm{in}^{2}$, the magnetic separation between the head and the disk must be reduced to approximately $3.5 \mathrm{~nm}$ (Wood 2000). One of the difficulties associated with low magnetic spacing, and, consequently, low flying height, is the increase in the number and severity of slider/disk contacts. Contacts between slider and disk are affected by intermolecular forces (Thornton and Bogy 2004) and adhesion forces (Ono et al. 2005). To reduce adhesion forces, slider designs with reduced contact area between slider and disk have been proposed (Zhu et al. 2000).

In this paper, the touch-down and take-off characteristics of pico-type magnetic recording sliders are investigated as a function of groove dimensions of DTR media. In addition, the touch-down and take-off characteristics of pico sliders are studied as a function of ambient pressure level and velocity for both smooth and discrete track media. Furthermore, contact start-stop (CSS) testing is performed to study the effect of grooves on the tribology of the head/disk interface. 


\section{Experimental setup and parameters}

Figure 1 shows a schematic of a typical "smooth" and "discrete" track recording head/disk interface. Figure 2 shows a schematic of the experimental setup consisting of a spin stand, a spin stand controller, a pressure regulator, a scanning laser Doppler vibrometer (S-LDV), and an acoustic emission (AE) sensor. The spin stand was placed in a sealed chamber to control the ambient pressure level. A S-LDV and AE sensors were connected to a data acquisition system to determine the flying characteristics of the head/disk interface.

Table 1 shows the types of DTR media used in our experimental work. To compare the touch-down and takeoff characteristics of discrete track media disks, we have used one smooth disk and three different DTR disks.

The air bearing design of the slider used throughout our investigation is shown in Fig. 3a. The slider was designed to fly at a flying height of $20 \mathrm{~nm}$ on a smooth disk at a velocity of $22 \mathrm{~m} / \mathrm{s}$. The flying height of the pico slider used in our experiments is also shown in Table 1 for the three DTR disks A, B, and C (based on Eq. 1). We observe that the flying height of the pico slider at the design velocity of $22 \mathrm{~m} / \mathrm{s}$ is $14 \mathrm{~nm}$ for disk A and $7.4 \mathrm{~nm}$ for disk B. The flying height at $22 \mathrm{~m} / \mathrm{s}$ is zero for disk C. Based on the flying height predictions for disks $\mathrm{A}, \mathrm{B}$, and $\mathrm{C}$, it is apparent that different touch-down velocities should be observed as a function of pressure level and type of disk used.

Figure $3 \mathrm{~b}$ shows the parameters of a typical DTR disk. The parameters of DTR media are groove depth $(d)$, groove width $(w)$, and track pitch $(p)$.

\section{Experimental results}

\subsection{Touch-down velocity for smooth and DTR media}

A S-LDV and an AE sensor were used to detect slider/disk contacts for both smooth and DTR media. For this measurement, the slider was first positioned at a radius of $39 \mathrm{~mm}$. Then, the velocity (RPM) was decreased slowly from $29.4 \mathrm{~m} / \mathrm{s}(7,200 \mathrm{RPM})$ in steps of $0.2 \mathrm{~m} / \mathrm{s}$ (50 RPM) until contact (touchdown) was observed. Figure 4 shows frequency spectra of the slider dynamics on a smooth disk

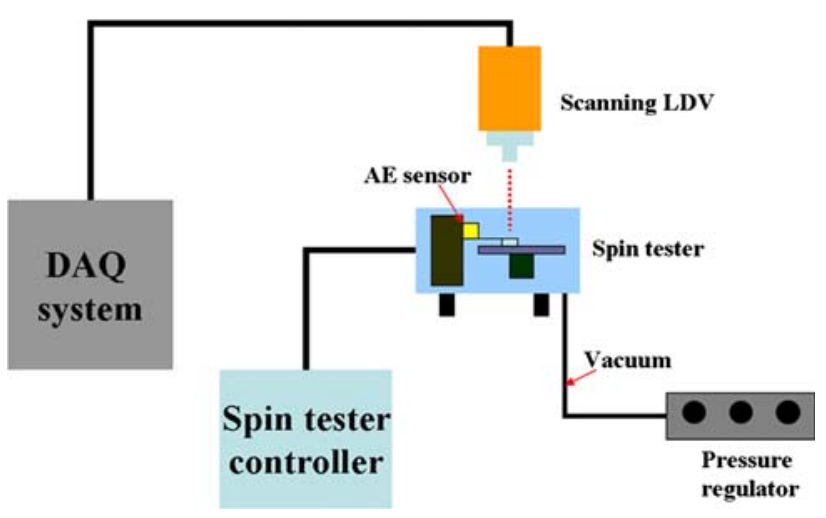

Fig. 2 Schematic of experimental setup

Table 1 Configurations of DTR media

\begin{tabular}{llll}
\hline & Disk A & Disk B & Disk C \\
\hline Groove depth $(d)[\mathrm{nm}]$ & 20 & 30 & 40 \\
Groove width $(w)[\mathrm{nm}]$ & 70 & 90 & 110 \\
Track pitch $(p)[\mathrm{nm}]$ & 210 & 200 & 200 \\
$w / p$ & 0.3 & 0.45 & 0.55 \\
Design flying height $[\mathrm{nm}]$ & 14 & 7.4 & 0 \\
\hline
\end{tabular}

for decreasing velocity. We observe that for velocities from $29.4 \mathrm{~m} / \mathrm{s}$ (7,200 RPM) to $3.3 \mathrm{~m} / \mathrm{s}$ (800 RPM), contacts are absent, i.e., the slider is flying. However, touch-down suddenly occurred at $3.1 \mathrm{~m} / \mathrm{s}$ (750 RPM). Now, both the S-LDV and the AE signal show large variations in amplitude with frequency, as can be seen from Fig. 4a,b, indicating contacts, i.e., touchdown. Figure 5 shows frequency spectra of slider dynamics on disk A. At velocities above $3.7 \mathrm{~m} / \mathrm{s}$ (900 RPM), touch-down is absent. However, if the velocity is decreased below $3.7 \mathrm{~m} / \mathrm{s}$, touch-down occurs at $3.5 \mathrm{~m} / \mathrm{s}$ (850 RPM), as can be seen from Fig. 5. In the case of disk B (Fig. 6), a few contacts were detected already at $8.2 \mathrm{~m} / \mathrm{s}(2,000 \mathrm{RPM})$, but touch-down of the slider occurred at $7.1 \mathrm{~m} / \mathrm{s}(1,750 \mathrm{RPM})$.

Figure 7 shows a summary of the touch-down velocity for the smooth and DTR media under investigation. For DTR disks A and B, touch-down is observed to occur at higher disk velocities than for the smooth disk. For disk C, contact occurred at all velocities throughout the experiment, i.e., the slider did not fly on disk $\mathrm{C}$ at all, not even at the highest velocity of $29.4 \mathrm{~m} / \mathrm{s}$ (7,200 RPM). Comparing
Fig. 1 Schematic of smooth disk (a) and DTR disk (b)
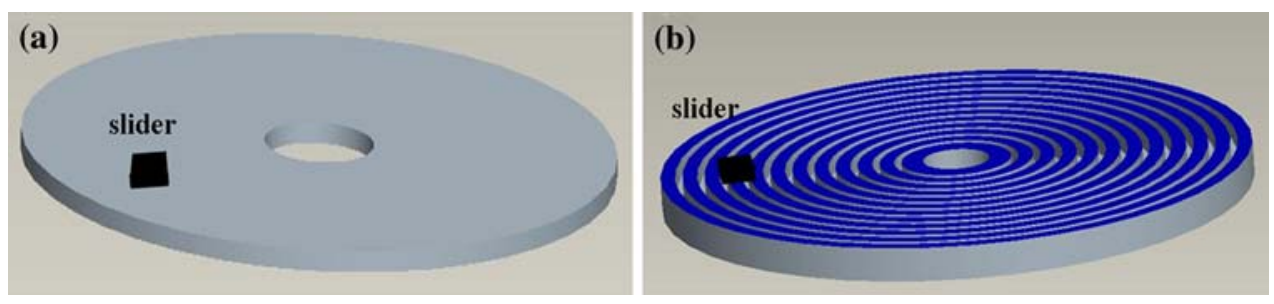
Fig. 3 Air bearing design of pico slider used in experiments (a) and parameters of DTR media (b) (a)

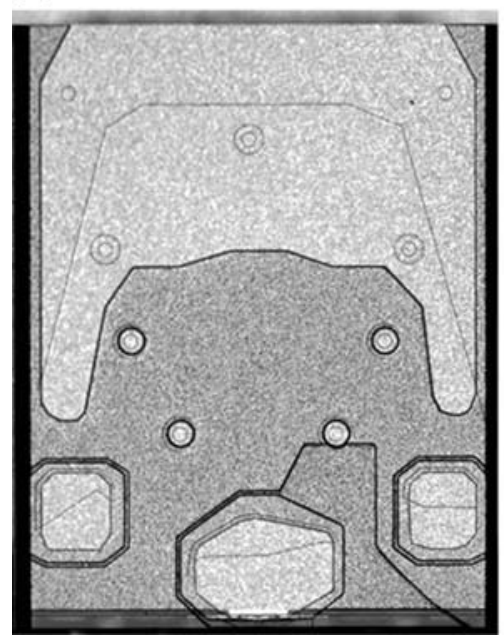

(b)

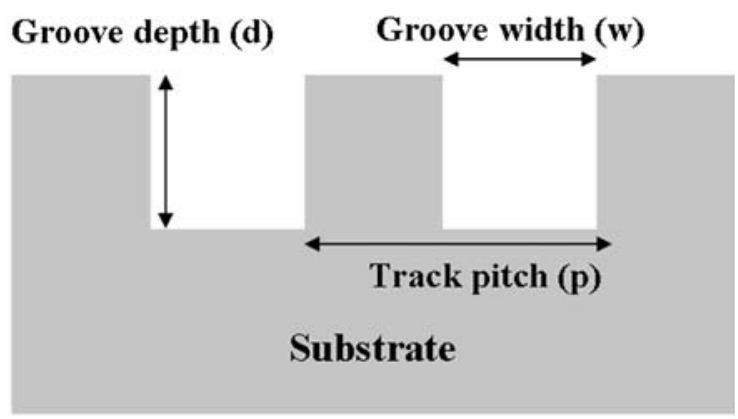

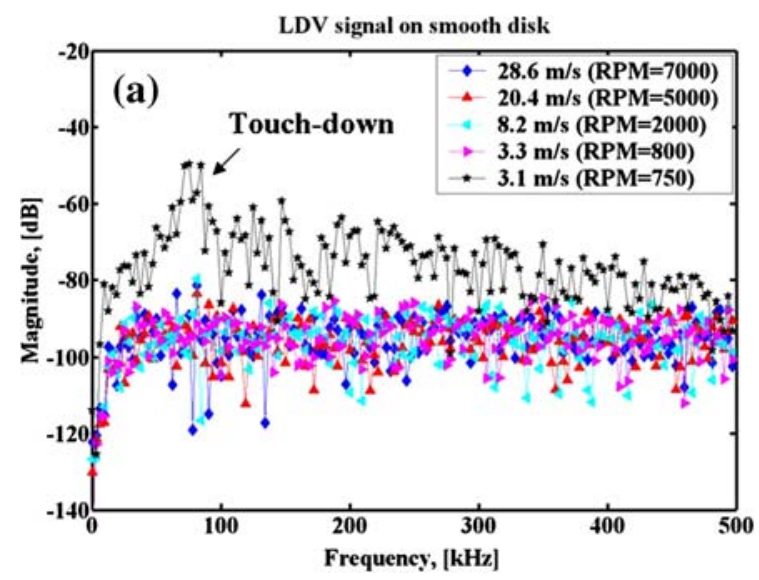

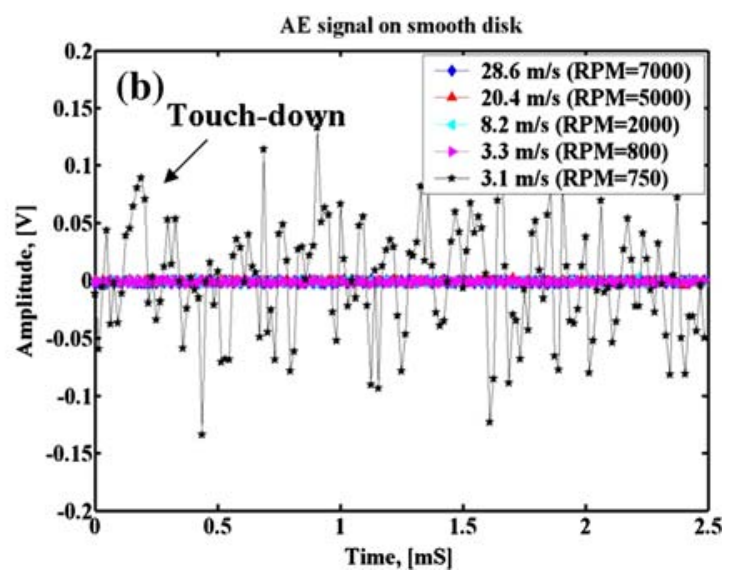

Fig. 4 Frequency spectra of disk velocity for smooth disk. a S-LDV signal. b AE signal
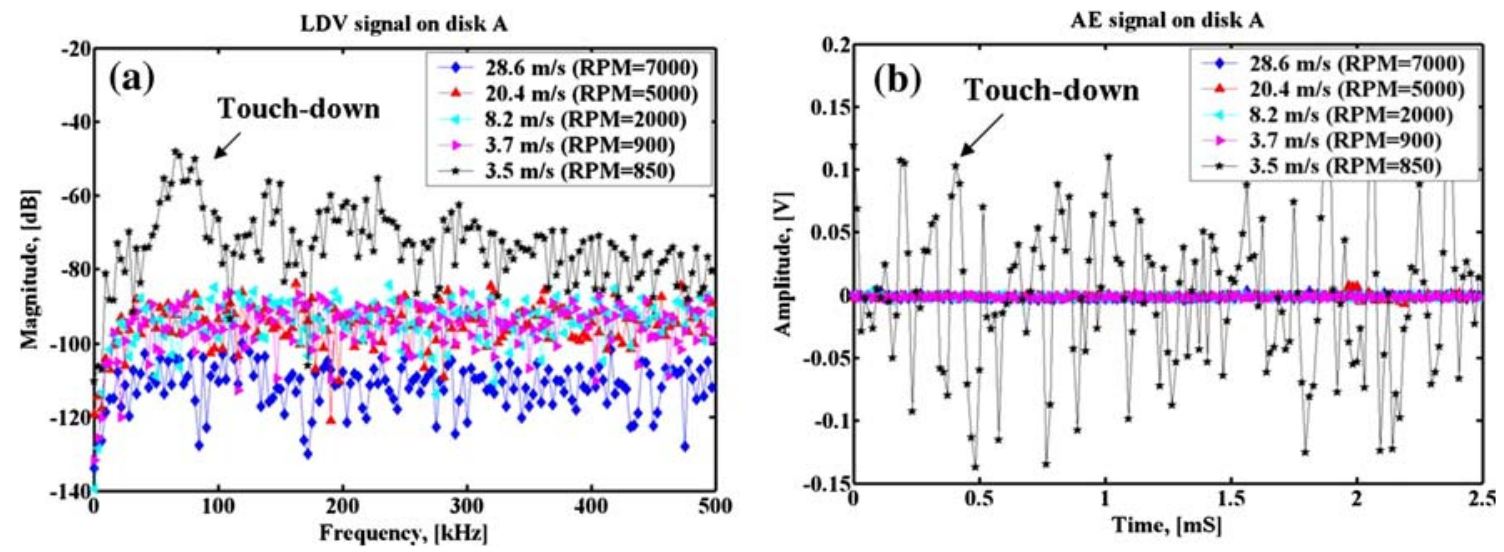

Fig. 5 Frequency spectra of disk velocity for disk A. a S-LDV signal. b AE signal

the touch-down velocity of disk A with the touch-down velocity of the smooth disk, we observe that the difference is small. On the other hand, a much larger difference is observed between disk $\mathrm{B}$ and the smooth disk or between disk A and B. Clearly, this difference in the touch-down velocity for disk $A$ and $B$ is a function of the nominal flying height of the slider on disk $A$ and $B$. In particular, using the flying height data from Table 1, we see that the design flying height of the pico slider for disk A is $14.0 \mathrm{~nm}$, while it is $7.4 \mathrm{~nm}$ for disk B. Thus, the slider flies lower on disk 

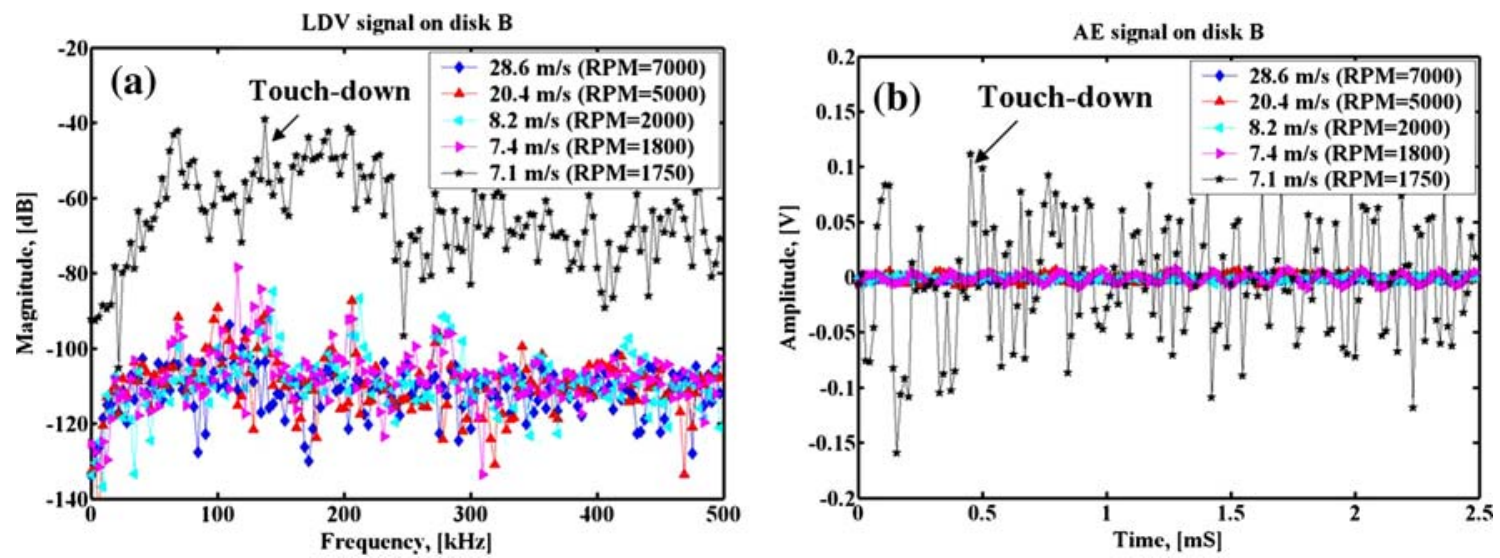

Fig. 6 Frequency spectra of disk velocity for disk B. a S-LDV signal. b AE signal

B than on disk A, and contacts or "touch-down" is likely to occur at a higher velocity on disk B than on disk A.

\subsection{Investigation of touch-down and take-off behavior as a function of ambient pressure for smooth and DTR media}

For this test, the slider was positioned at a disk radius of $37 \mathrm{~mm}$ and the velocity was kept at $27.9 \mathrm{~m} / \mathrm{s}$ (7,200 RPM). To detect touch-down, the ambient pressure level was decreased in small increments until severe slider/disk contacts were observed. After touchdown, the ambient pressure was increased until contacts between the slider and the disk disappeared.

Figure 8a, b shows AE signals of touch-down and takeoff, respectively, as a function of normalized ambient pressure level $P^{*}=p_{\text {ambient }} / p_{\text {atmospheric }}$ for flying on a smooth disk. We observe that contacts are absent for normalized pressure levels $P^{*}>0.4$. However, if the pressure level $P^{*}$ was decreased below $P^{*}=0.4$, severe contacts (i.e., touch-down) occurred at $P^{*}=0.33$. In Fig. $8 \mathrm{~b}$, the reverse procedure is followed, i.e., the pressure is increased in small increments above the touch-down pressure of $P^{*}=0.33$. We observe that a normalized pressure of $P^{*}=0.38$ is needed before flying is re-established. This

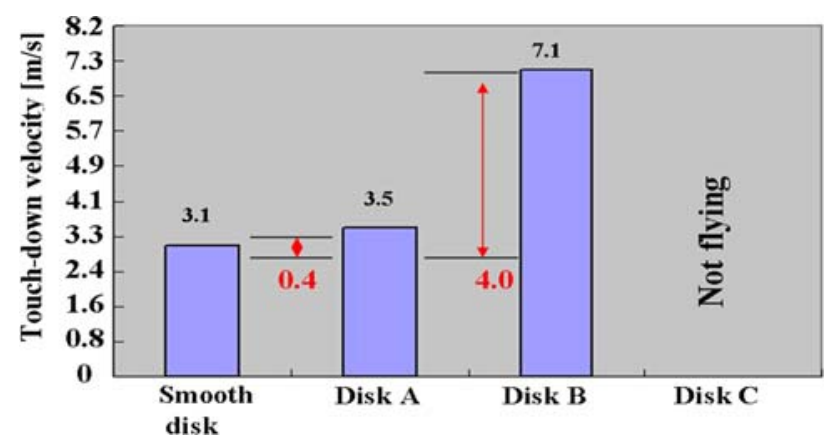

Fig. 7 Touch-down velocity for smooth and DTR disks difference in touch-down and take-off pressure is defined in this paper as "pressure hysteresis".

Figure 9 shows AE signals of normalized touch-down and take-off ambient pressure $P^{*}$ for disk A. In this case, touch-down of the slider was observed at $P^{*}=0.39$ while take-off occurred at $P^{*}=0.66$. In the case of disk B, touchdown and take-off occurred at $P^{*}=0.67$ and $P^{*}=0.98$, respectively, as shown in Fig. 10. Figure 11 gives a summary of the pressure data for the normalized touch-down pressure $P^{*}$ for the head/disk combinations investigated. For discrete track disks A and B, touch-down was found to occur at a higher normalized pressure level $P^{*}$ compared to the touch-down pressure on a smooth disk. Again, the slider was not flying on disk C. Similar to the results shown in Fig. 7, the difference in the normalized touch-down pressure for the smooth disk and disk B was much larger than the difference between the smooth disk and disk A. Again, this result is to be expected based on the design flying height data for the pico slider used on the two types of disks (see also Table 1).

Figure 12 shows the hysteresis between normalized touch-down and take-off pressure on both the smooth disk and the three DTR disks at a velocity of $27.9 \mathrm{~m} / \mathrm{s}$. For the smooth disk, the hysteresis between touch-down and takeoff pressure is small. However, both DTR disks A and B show hysteresis between touch-down and take-off pressure, the difference being much larger for disk B than for disk A. Again, the reason for this behavior is related to the nominal flying height of the slider on disk A and B (see Table 1). No hysteresis occurred for disk $\mathrm{C}$ since the slider was not flying on this disk at all.

\subsection{Contact start-stop test}

To evaluate the effect of take-off velocity on the tribological performance of the head/disk interface for smooth and DTR media, CSS tests were conducted at a radius of $35 \mathrm{~mm}$ and a skew angle of zero degrees. 

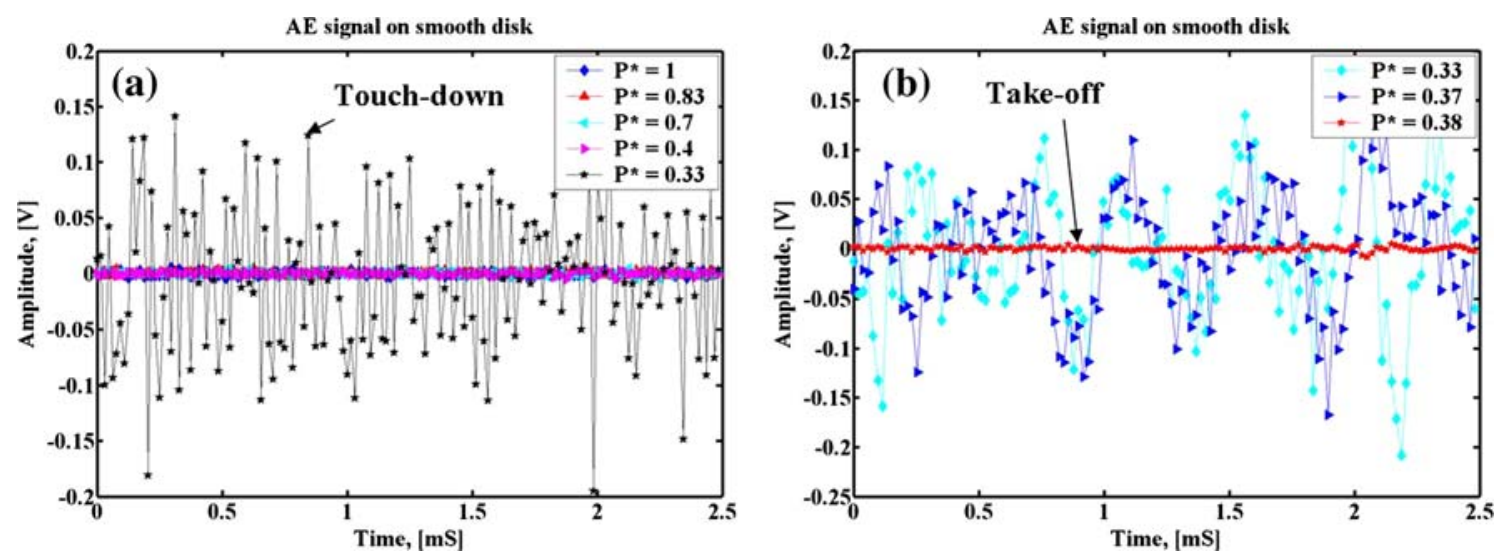

Fig. 8 Normalized pressure $P^{*}$ at touch-down and take-off for a smooth disk at a velocity of $27.9 \mathrm{~m} / \mathrm{s}$. a Touch-down signal. b Take-off signal
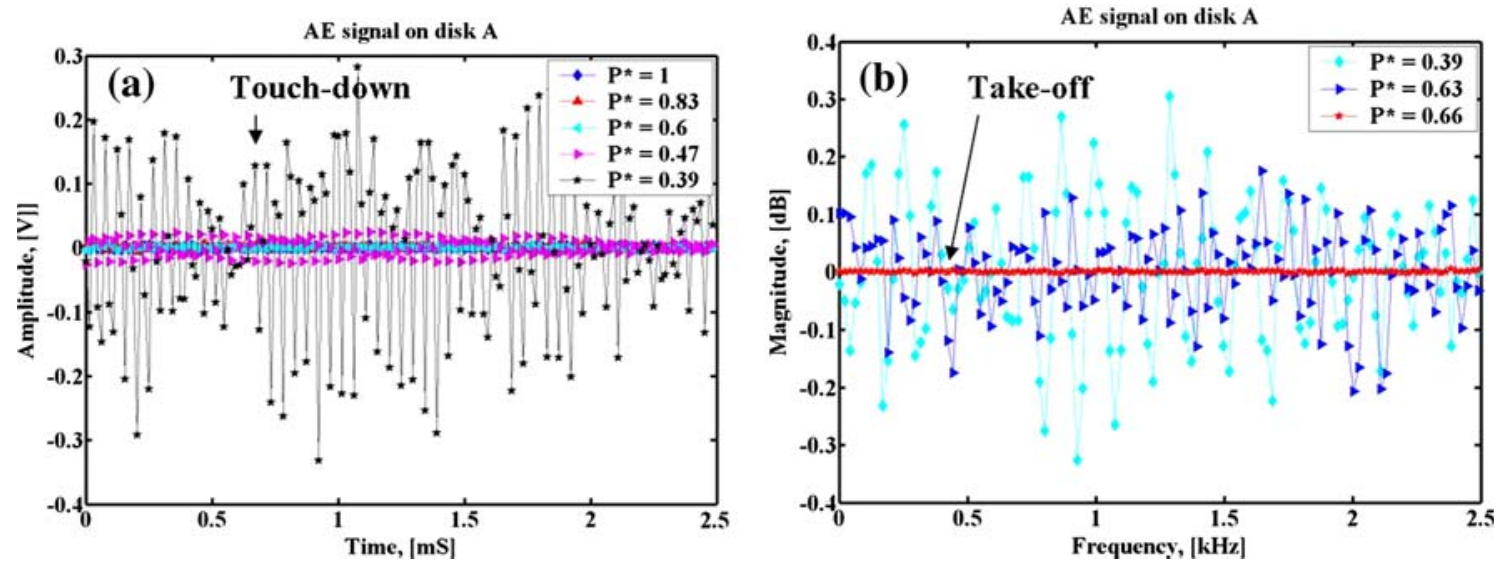

Fig. 9 Normalized pressure $P^{*}$ at touch-down and take-off for disk A at a velocity of $27.9 \mathrm{~m} / \mathrm{s}$. a Touch-down signal. b Take-off signal
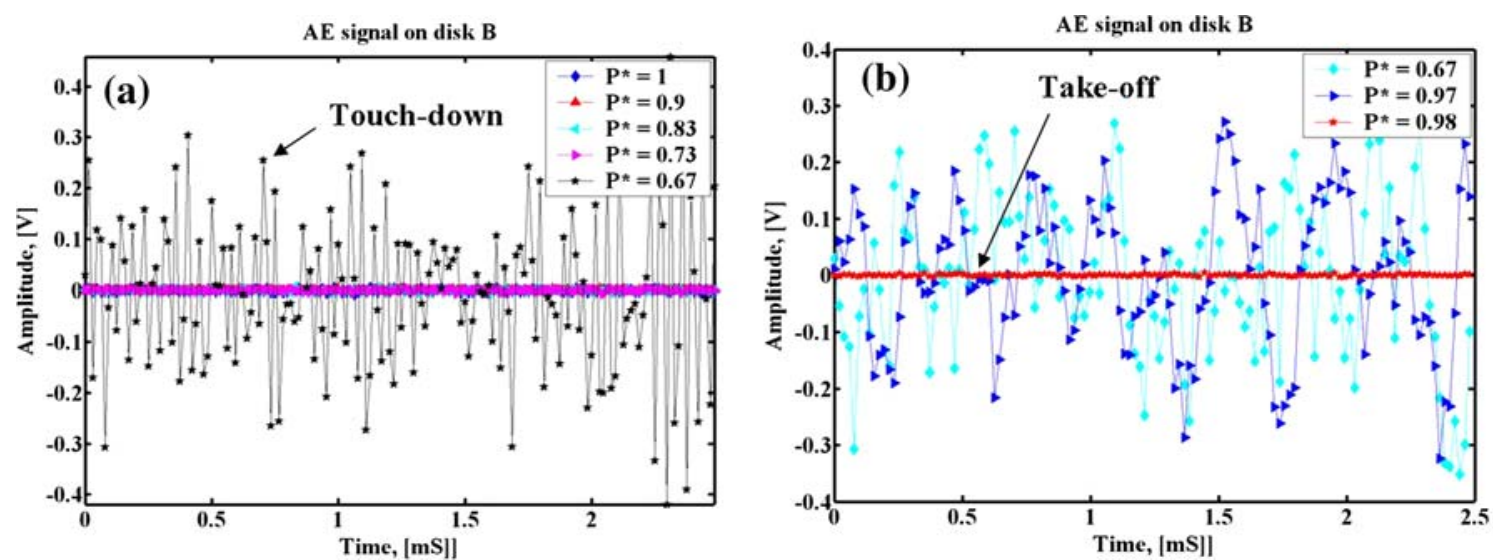

Fig. 10 Normalized pressure $P^{*}$ at touch-down and take-off for disk B at a velocity of $27.9 \mathrm{~m} / \mathrm{s}$. a Touch-down signal. b Take-off signal

Figure 13 shows the velocity profile of a typical CSS test performed in this investigation. In the first 3.5 seconds, the velocity was increased to $26.4 \mathrm{~m} / \mathrm{s}(7,200 \mathrm{RPM})$. The velocity was kept constant at this level for 5-s, and was then decreased linearly with time to zero in about $3.5 \mathrm{~s}$.

Figure 14 shows a summary of the velocities at which continuous flying of the slider was established during start/ stop testing. We observe that the velocity, at which flying is first observed, is lower on the smooth disk than on the DTR disks.

For disks $\mathrm{A}$ and $\mathrm{B}$, a higher disk velocity is required to establish hydrodynamic flying than for the smooth disk. As expected from the data in Figs. 7 and 11, the velocity at which flying is established on disk B is much larger than 


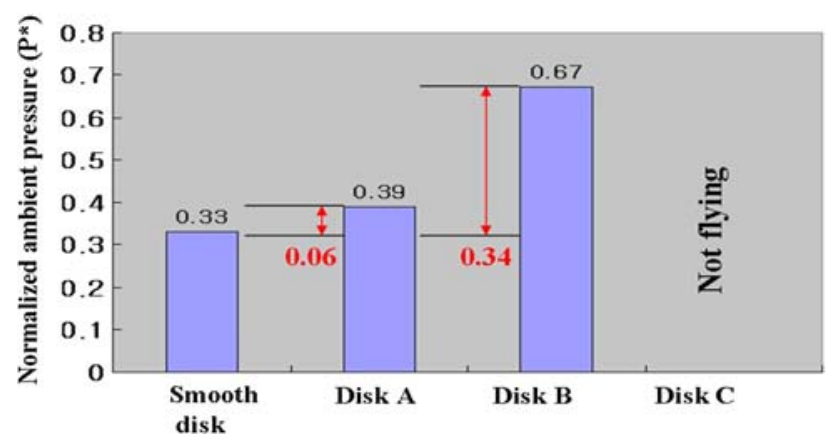

Fig. 11 Normalized ambient touch-down pressure $P^{*}$ for smooth and discrete track recording disks at a velocity of $27.9 \mathrm{~m} / \mathrm{s}$

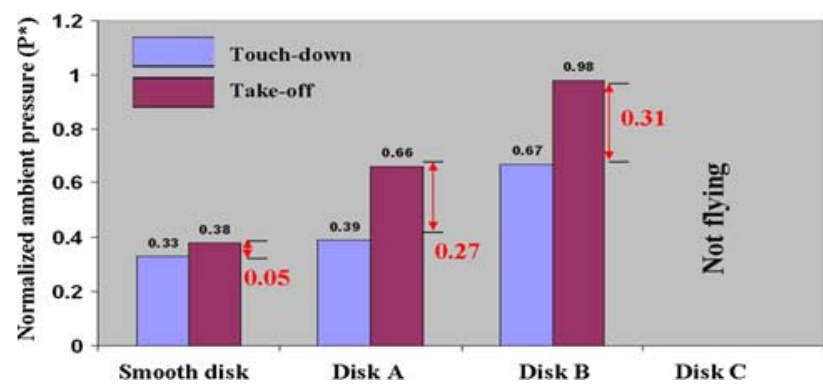

Fig. 12 Touch-down and take-off pressure hysteresis on a smooth disk and DTR media at a velocity of $27.6 \mathrm{~m} / \mathrm{s}$

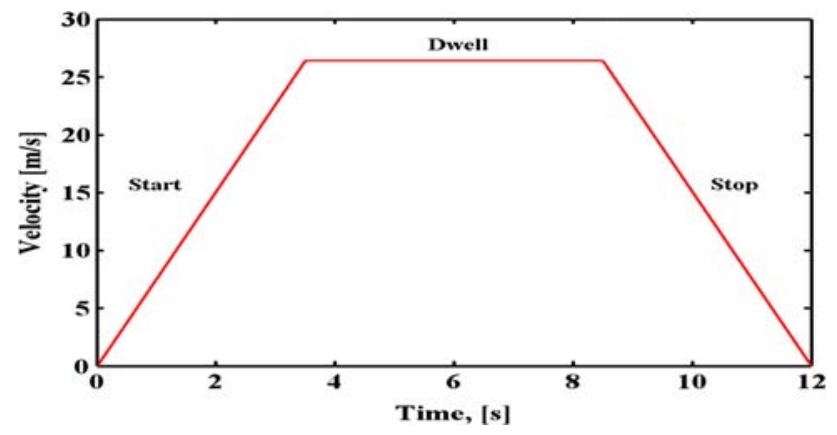

Fig. 13 Velocity profile for contact start-stop test

the velocity when flying is established on disk A or the smooth disk. This, again, is a direct function of the lower design flying height of the slider on disk B versus that on disk A (see Table 1). It is interesting to note that flying of the slider was not observed on disk $\mathrm{C}$.

\section{Summary and conclusions}

The touch-down characteristics of a pico-type magnetic recording sliders was investigated on DTR media as a function of disk velocity and ambient pressure level. Using the same slider design, we observed that the touch-down velocity was higher for flying of the slider on DTR media

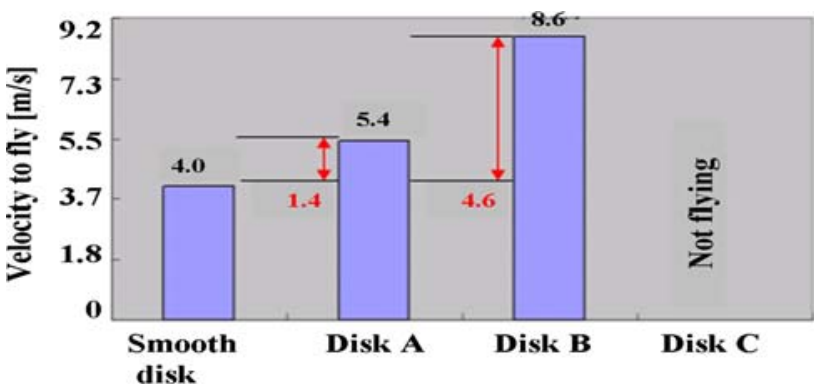

Fig. 14 Critical velocity for flying of slider on a smooth disk and discrete track recording disks during start/stop testing

than on smooth media. Keeping the velocity constant and reducing the ambient pressure level for the same slider, we observed that touch-down occurred at a higher pressure level on DTR media than on smooth media. This effect is related to the nominal flying height of the slider on smooth and discrete track media. A well-defined hysteresis occurred between touch-down and take-off velocity as well as touch-down and take-off pressure, depending on the design flying height of the slider and the characteristics of the discrete track media. The results for touch-down and take-off behavior of the slider used in this study are in qualitative agreement with the data from start-stop investigations.

Acknowledgment The authors would like to thank Western Digital Corporation for providing the components used in this study.

Open Access This article is distributed under the terms of the Creative Commons Attribution Noncommercial License which permits any noncommercial use, distribution, and reproduction in any medium, provided the original author(s) and source are credited.

\section{References}

Duwensee M, Suzuki S, Lin J, Wachenschwanz D, Talke FE (2006) Air bearing simulation of discrete track recording media. IEEE Trans Mag 42(10):2489-2491

Duwensee M, Suzuki S, Lin J, Wachenschwanz D, Talke FE (2007) Simulation of the head disk interface for discrete track media. Microsyst Technol 13:1023-1030

Ono K, Yamane M, Yamaura H (2005) Experimental and analytical study of bouncing vibrations of a flying head slider in a nearcontact regime. Trans ASME 127:376-386

Thornton BH, Bogy DB (2004) A parametric study of head-disk interface instability due to intermolecular forces. IEEE Trans Magn 40(1):337-344

Wachenschwanz D et al (2005) Design of a manufacturable discrete track recording medium. IEEE Trans Magn 41(2):670-675

Wood R (2000) The feasibility of magnetic recording at 1 terabit per square inch. IEEE Trans Magn 36:36-42

Zhu Y-L, Liu B, Hua W, Sheng G (2000) A study of interface dynamics for stiction-free slider and super-smooth disk. J Appl Phys 87:6149-6151 